\title{
Modeling Correlation of Quantized Noise and Periodic Signals
}

\author{
André B. J. Kokkeler and André W. Gunst
}

\begin{abstract}
A model for determining the cross-correlation function of partially correlated noise is presented. In this model a strong interferer is included and represented by a periodic signal common to both channels of the correlator. A general expression for the correlation function is deduced and verified. The power spectrum of a calculated correlation function is compared with a simulation. The results presented in this paper form a base for the design of modern multibit correlators. These are part of future generation radio astronomy receivers which increasingly have to cope with man-made interfering signals.
\end{abstract}

Index Terms-A/D converters, correlation, dither, quantization.

\section{INTRODUCTION}

W ITHIN radio astronomy, the radio window of the earth's atmosphere (roughly from $20 \mathrm{MHz}$ to $30 \mathrm{GHz}$ ) is used for observations of celestial objects. In general, the power of the radiation received from these objects is below the power of the noise produced in the analog parts of a radio astronomy receiver (RA receiver). A technique to reduce the effects of the noise is to cross-correlate signals within a multiple-telescope RA receiver. This way, signals up to $70 \mathrm{~dB}$ below the noise level can be detected.

Interference, common to all telescopes, remains visible after cross-correlation. As a result of the nonlinearity of the analog-to-digital converter (A/D converter), harmonics of the interference are present as well, contaminating the whole spectrum. RA receivers have been designed to have sufficient selectivity in the analog paths to the $\mathrm{A} / \mathrm{D}$ converters to remove interference. Because of the increasing bandwidth of the A/D converters and the growing use of the electro magnetic spectrum, the condition that the signals at the input of the $\mathrm{A} / \mathrm{D}$ converters do not contain interference is no longer satisfied. For the design of modern RA receivers, it is therefore necessary to be able to analyze the effect of narrowband interference for different resolutions of the A/D converter.

The effects of quantization for the case no interference is present is studied in [1] and [2]. This work is strongly related to the analysis of quantizing systems using nonsubtractive dither [3], where the measured correlation coefficients equal the nonsubtractive dithered second order moments.

Manuscript received December 24, 2003; revised February 15, 2004. The associate editor coordinating the review of this manuscript and approving it for publication was Dr. Xiang-Gen Xia.

A. B. J. Kokkeler is with the Department of Electrical Engineering, Computer Science and Mathematics, University of Twente, 7500 AE Enschede, The Netherlands (e-mail: a.b.j.kokkeler@utwente.nl).

A. W. Gunst is with the Netherlands Foundation for Research in Astronomy (ASTRON), 7990 AA Dwingeloo, The Netherlands (e-mail: gunst@astron.nl). Digital Object Identifier 10.1109/LSP.2004.835481

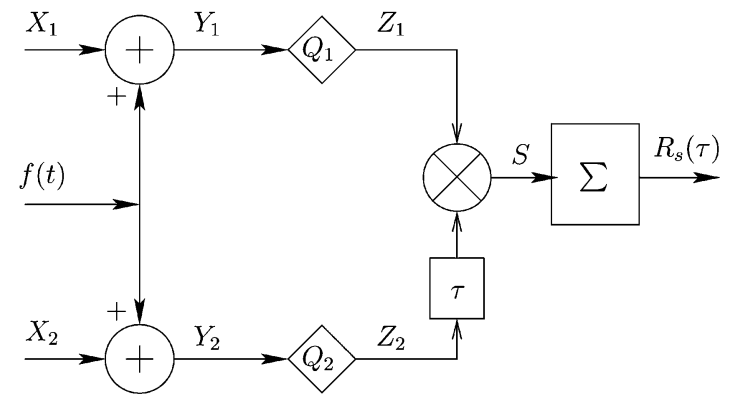

Fig. 1. A general correlator model.

The cross-correlation of noise and periodic signals has been studied in [4] but only in case of extreme clipping and a small signal-to-noise ratio (SNR). Multibit quantization and arbitrary SNRs are not considered. Multibit quantization of sinusoidal signals has been studied intensively (see [5] and [6]), but the developed theory is not applicable to correlation configurations.

Within this paper, a generic model of a cross-correlator for different types of input signals is given. A novel general expression for the measured cross-correlation function in case of multibit quantization of a signal containing noise and a periodic signal is found. This expression is elaborated in case the periodic signal is a sinusoidal signal. Only quantization effects are considered. We check the expression against known results and conclude with an example of a calculated and simulated spectrum.

\section{A General Correlator Model}

\section{A. The Correlation Function}

The correlation function of a sine wave plus noise after extreme clipping has been determined in [4]. Based on that analysis, a general correlator model is presented in Fig. 1.

There are two wide-sense stationary noise signals $X_{1}$ and $X_{2}$. A periodic signal $f(t)$ is added to both $X_{1}$ and $X_{2}$. The resulting signals $Y_{1}$ and $Y_{2}$ are quantized by $Q_{1}$ and $Q_{2}$, respectively, and cross -correlated. Because $X_{1}$ and $X_{2}$ are wide-sense stationary, the noise inputs can be described with a bivariate normal distribution. Without loss of generality it is assumed that $\mu=0$ and $\sigma=1$ for both $X_{1}$ and $X_{2}$. If we consider $X_{1}$ and $X_{2}$ at the respective times $t$ and $t-\tau$, the correlation equals $\rho(\tau)$. $Y_{1}$ and $Y_{2}$ are quantized by an N-bit mid-riser quantizer with unit step size

$$
Q(x)=\left(\sum_{i=-2^{N-1}+1}^{2^{N-1}-1} \Phi(g x-i)\right)-2^{N-1}+\frac{1}{2} .
$$


$\Phi$ is Heaviside's step function and $g$ determines the granularity of the quantization process. After quantization, $Z_{1}$ and $Z_{2}$ are multiplied and averaged. We define the time-dependent correlation function $R(t, \tau)$ as the ensemble average of $Z_{1}(t)$ and $Z_{2}(t-\tau)$

$$
R(t, \tau)=\left\langle Z_{1}(t) \cdot Z_{2}(t-\tau)\right\rangle
$$

The correlation function $R_{s}(\tau)$ equals the time average of $R(t, \tau)$ over one cycle of the periodic signal with period $T_{p}$ (see [4]). If the signal after multiplication is sampled and integrated, $R_{s}(\tau)$ is described by

$$
R_{s}(\tau)=\frac{1}{T_{p}} \sum_{t=0}^{T_{p}-1} R(t, \tau)
$$

\section{B. Splitting the Noise Sources}

As is done in [7], the two signals $X_{1}$ and $X_{2}$ can be modeled by three uncorrelated Gaussian noise sources $N_{0}, N_{1}$, and $N_{2}$, all having $\mu=0$ and $\sigma=1$

$$
\begin{aligned}
& X_{1}=\sqrt{1-|\rho(\tau)|} N_{1}+\sqrt{|\rho(\tau)|} N_{0} \\
& X_{2}=\sqrt{1-|\rho(\tau)|} N_{2}+\operatorname{sign}(\rho(\tau)) \sqrt{|\rho(\tau)|} N_{0} .
\end{aligned}
$$

The $\operatorname{sign}(\rho)$ operator is defined as

$$
\operatorname{sign}(\rho)= \begin{cases}-1, & \text { if } \rho<0 \\ 1, & \text { otherwise. }\end{cases}
$$

The periodic signal $f(t)$ is added to both $X_{1}$ and $X_{2}$. The components due to $N_{0}$ and $f$ are defined as displacements

$$
\begin{aligned}
& \delta_{1}\left(n_{0}, t, \tau\right)=\sqrt{|\rho(\tau)|} n_{0}+f(t) \\
& \delta_{2}\left(n_{0}, t, \tau\right)=\operatorname{sign}(\rho(\tau)) \sqrt{|\rho(\tau)|} n_{0}+f(t-\tau) .
\end{aligned}
$$

This leads to the following expressions for $Y_{1}$ and $Y_{2}$

$$
\begin{aligned}
& Y_{1}=\sqrt{1-|\rho(\tau)|} N_{1}+\delta_{1}\left(n_{0}, t, \tau\right) \\
& Y_{2}=\sqrt{1-|\rho(\tau)|} N_{2}+\delta_{2}\left(n_{0}, t, \tau\right) .
\end{aligned}
$$

For both quantizers, the input is the sum of two stochastic signals. Signals due to $N_{1}$ and $N_{2}$ are uncorrelated and have power $1-|\rho(\tau)|$, while the displacements $\delta_{1}$ and $\delta_{2}$ depend on $N_{0}$. The power of $N_{0}$ equals 1 . The time-dependent correlation function $R(t, \tau)$ can be written as the ensemble average over the three noise sources $N_{0}, N_{1}$ and $N_{2}$

$$
\begin{aligned}
R(t, \tau)= & \int_{-\infty}^{\infty} \int_{-\infty}^{\infty} \int_{-\infty}^{\infty} f_{N_{0}}\left(n_{0}\right) f_{N}\left(n_{1}\right) f_{N}\left(n_{2}\right) \\
& \cdot Q\left(n_{1}+\delta_{1}\left(n_{0}, t, \tau\right)\right) \cdot Q\left(n_{2}+\delta_{2}\left(n_{0}, t, \tau\right)\right) \\
& \cdot d n_{2} d n_{1} d n_{0}
\end{aligned}
$$

where

$$
\begin{aligned}
f_{N_{0}}\left(n_{0}\right) & =\frac{1}{\sqrt{2 \pi}} e^{-\frac{1}{2} n_{0}^{2}} \\
f_{N}(n) & =\frac{1}{\sqrt{2 \pi(1-|\rho(\tau)|)}} e^{-\frac{1}{2}\left(\frac{n}{\sqrt{1-|\rho(\tau)|}}\right)^{2}} .
\end{aligned}
$$

By rearranging this formula, two gain functions $G_{1}$ and $G_{2}$ can be defined

$$
R(t, \tau)=\int_{-\infty}^{\infty} f_{N_{0}}\left(n_{0}\right) \cdot G_{1}\left(n_{0}, t, \tau\right) \cdot G_{2}\left(n_{0}, t, \tau\right) \cdot d n_{0}
$$

where

$$
\begin{aligned}
& G_{1}\left(n_{0}, t, \tau\right)=\int_{-\infty}^{\infty} f_{N}\left(n_{1}\right) \cdot Q\left(n_{1}+\delta_{1}\left(n_{0}, t, \tau\right)\right) \cdot d n_{1}(15) \\
& G_{2}\left(n_{0}, t, \tau\right)=\int_{-\infty}^{\infty} f_{N}\left(n_{2}\right) \cdot Q\left(n_{2}+\delta_{2}\left(n_{0}, t, \tau\right)\right) \cdot d n_{2}(16)
\end{aligned}
$$

\section{Elaborating $G_{1}$}

The quantization staircase consists of an ideal transfer function and an error (see [6])

$$
Q(x)=g x+\sum_{m=1}^{\infty} \frac{1}{m \pi} \sin (2 m \pi g x) .
$$

Using this in (15), using standard rules for integration and trigonometry, and inserting (7), the expression for $G_{1}$ can be written as

$$
\begin{aligned}
G_{1}\left(n_{0}, t, \tau\right)= & g f(t)+g \sqrt{|\rho(\tau)|} n_{0} \\
& +\sum_{m=1}^{\infty} \frac{1}{m \pi} e^{-2 \pi^{2} m g(1-|\rho(\tau)|)} \\
& \cdot\left[\left(\cos \left(2 \pi m g \sqrt{|\rho(\tau)|} n_{0}\right) \cdot \sin (2 \pi m g f(t))\right)\right. \\
& \left.+\left(\sin \left(2 \pi m g \sqrt{\rho(\tau) \mid} n_{0}\right) \cdot \cos \mid(2 \pi m g f(t))\right)\right] .
\end{aligned}
$$

A similar expression can be found for $G_{2}$, where $\sqrt{|\rho(\tau)|}$ is replaced by $\operatorname{sign}(\rho(\tau)) \sqrt{|\rho(\tau)|}$ and $f(t)$ by $f(t-\tau)$.

\section{Final Expression}

Using the expressions for $G_{1}$ and $G_{2}, R(t, \tau)$ (see (14)) can be calculated. In Appendix A, an expression is given for any periodic signal $f(t)$. If the input signal is defined as $f(t)=$ $a \sin (\Psi(t))$, the following expressions can be used to rewrite the equation in Appendix A

$$
\begin{aligned}
\sin (z \sin (\Psi))= & 2 \sum_{p=1, \text { odd }}^{\infty} J_{p}(z) \sin (p \Psi) \\
\cos (z \sin (\Psi))= & J_{0}(z) \cos (\Psi) \\
& +2 \sum_{p=2, \text { even }}^{\infty} J_{p}(z) \cos (p \Psi) .
\end{aligned}
$$

$J_{p}(z)$ is the $p$ th-order Bessel function of the first kind. $R_{s}(\tau)$ can then be expressed as

$$
\begin{aligned}
R_{s}(\tau)= & \left(1+\epsilon_{\rho}\right) g^{2} \rho(\tau)+\left(1+\epsilon_{f}\right) \frac{g^{2} a^{2}}{2} \cos (\Psi(\tau)) \\
& +\frac{1}{2} \epsilon_{p=0}^{e}+\sum_{p=1, \text { odd }}^{\infty} \epsilon_{p}^{o} \cos (p \Psi(\tau)) \\
& +\sum_{p=2, \text { even }}^{\infty} \epsilon_{p}^{e} \cos (p \Psi(\tau))
\end{aligned}
$$


where

$$
\begin{aligned}
& \epsilon_{\rho}= 4 \sum_{m=1}^{\infty} J_{0}(2 \pi m g a) e^{-2 \pi^{2} m^{2} g^{2}} \\
& \epsilon_{f}= \frac{4}{g a} \sum_{m=1}^{\infty} \frac{1}{m \pi} J_{1}(2 \pi m g a) e^{-2 \pi^{2} m^{2} g^{2}} \\
& \epsilon_{p}^{e}= \sum_{m_{1}=1}^{\infty} \sum_{m_{2}=1}^{\infty} \frac{2}{m_{1} m_{2} \pi^{2}} J_{p}\left(2 \pi m_{1} g a\right) J_{p}\left(2 \pi m_{2} g a\right) \\
& \times e^{-2 \pi^{2}\left(m_{1}^{2}+m_{2}^{2}\right) g^{2}} \cdot \sinh \left(4 \pi^{2} g^{2} m_{1} m_{2} \rho(\tau)\right), \\
& \epsilon_{p}^{o}= \sum_{m_{1}=1}^{\infty} \sum_{m_{2}=1}^{\infty} \frac{2}{m_{1} m_{2} \pi^{2}} J_{p}\left(2 \pi m_{1} g a\right) J_{p}\left(2 \pi m_{2} g a\right) \\
& \times e^{-2 \pi^{2}\left(m_{1}^{2}+m_{2}^{2}\right) g^{2}} \cdot \cosh \left(4 \pi^{2} g^{2} m_{1} m_{2} \rho(\tau)\right), \\
& p=1,3,5 \ldots
\end{aligned}
$$

An interpretation of the distortion coefficients is that $\epsilon_{\rho}$ and $\epsilon_{p=0}^{e}$ represent the distortion of the correlated noise part. $\epsilon_{f}$ represents the distortion of the sinusoidal signal. $\epsilon_{p}^{e}, p \neq 0$ and $\epsilon_{p}^{o}$ represent a combined effect.

An alternative approach to calculating the cross-correlation function in case no interference is present $(f(t)=0, \forall t)$, is to use the second-order joint-moment given as

$$
E\left[Z_{1}, Z_{2}\right]=\left(1+\delta\left(a_{1}, a_{2}\right)\right) E\left[Y_{1}, Y_{2}\right]+\Delta \mu_{12}\left(a_{1}, a_{2}\right)
$$

For the elaborated version of this formula, we refer to [8]. The gain of the system is influenced by $\delta$ and the error is reflected by $\Delta \mu_{12}$. Both $\delta$ and $\Delta \mu_{12}$ are defined in [8] and depend on $a_{1}$ and $a_{2}$, the expected values of $Y_{1}$ and $Y_{2}$, respectively. The more intuitive analysis presented above equals the analysis of the second-order joint moments when $a_{1}=a_{2}=0$.

\section{CASE Studies}

\section{A. Single-Bit Quantization of Noise}

In the case of single-bit quantization of noise, there is a one-to-one relation between the correlation function of the signals before quantization $(\rho(\tau))$ and the correlation function after quantization $\left(R_{s}(\tau)\right)$. This relation is well known as the Van Vleck relation (see [9]). The Van Vleck relation used in the configuration described above equals

$$
R_{s}^{v v}(\tau)=\frac{1}{4} \cdot \frac{1}{2} \arcsin (\rho(\tau))
$$

where the additional factor $(1 / 4)$ is caused by the two possible output values of the A/D converter being - $(1 / 2)$ and $(1 / 2)$ instead of -1 and 1 . This Van Vleck relation should equal expression (21). For single-bit quantization of noise, the scaling factor before quantization is small $(g \ll 1)$ and the amplitude of the periodic signal is set to zero $(a=0)$. Using these values, expression (21) cannot simply be reduced to the Van Vleck relation. We therefore calculated $R_{s}^{v v}(\tau)$ and $R_{s}(\tau)$ separately and compared the results. For $R_{s}(\tau)$ the summations over $m, m_{1}$ and $m_{2}$ run from 1 to 50 . From the calculations it appears that the difference between $R_{s}(\tau)$ and $R_{s}^{v v}(\tau)$ is smaller than $1.610^{-2}$.
This difference becomes smaller if $m, m_{1}$ and $m_{2}$ run over a larger range. This illustrates that expression (21) is correct for single-bit quantization of noise.

\section{B. Multibit Quantization of a Sinusoidal Signal Without Noise}

This situation occurs if the noise powers before quantization (and after scaling with $g$ ) are small. This means that $g$ is small. The power of the sinusoidal signal $\left((1 / 2) g^{2} a^{2}\right)$ is nonzero. The measured correlation function then becomes

$$
\begin{gathered}
R_{s}(\tau) \approx\left(1+\epsilon_{f}\right) \frac{g^{2} a^{2}}{2} \cos (\Psi(\tau)) \\
+\sum_{p=1, \text { odd }}^{\infty} \epsilon_{p}^{o} \cos (p \Psi(\tau))
\end{gathered}
$$

where

$$
\begin{aligned}
\epsilon_{f} & \approx \frac{4}{g a} \sum_{m=1}^{\infty} \frac{1}{m \pi} J_{1}(2 \pi m g a) \\
\epsilon_{p}^{o} & \approx\left(\sum_{m=1}^{\infty} \frac{\sqrt{2}}{m \pi} J_{p}(2 \pi m g a)\right)^{2} .
\end{aligned}
$$

The amplitude of the fundamental of the sinusoidal signal $\cos (\Psi(\tau))$ becomes

$$
\frac{1}{2}\left(g a+\sum_{m=1}^{\infty} \frac{2}{m \pi} J_{1}(2 \pi m g a)\right)^{2} .
$$

For the harmonic signals $(p>1)$, the amplitudes of $\cos (p \Psi(\tau))$ equal $\epsilon_{p}^{o}$. The amplitudes found this way equal half the squared gain factors found in [6]. This is to be expected, since in [6], the output signal of a single A/D converter as a function of time is considered, while in this document we are interested in the correlation function of this signal giving power.

\section{An Example of a Spectrum}

As an example, we calculated the cross-correlation of a 2.5 LSB sinusoidal signal with (1/4) LSB root mean square (rms) noise at the quantization staircases using (21). The noise at the two A/D converters is partially correlated with $\rho(0)=(1 / 2)$. We also simulated the set-up where we used pseudorandom noise sources to model $N_{0}, N_{1}$, and $N_{2}$. The cross-correlation function is used to determine the cross-power spectral density. The results of both the calculation and the simulation are given in Fig. 2.

The bandwidth of the correlated noise signal $\left(N_{0}\right)$ is $(1 / 32)$ nd of the sampling frequency and the frequency of the sinusoidal signal is $(1 / 16)$ th of the sampling frequency. It is chosen this way in order to illustrate the effect that the noise spectrum is repeated around the even harmonics of the sinusoidal signal. The bandwidth of the uncorrelated noise sources $N_{1}$ and $N_{2}$ is half the sampling frequency.

A small difference between the simulated and calculated spectrum can be observed. This is due to the fact that for practical reasons, sampling before multiplication is used in the simulation model. This sampling causes aliasing on both inputs of the multiplier. Amplitudes of sinusoidal signals with 


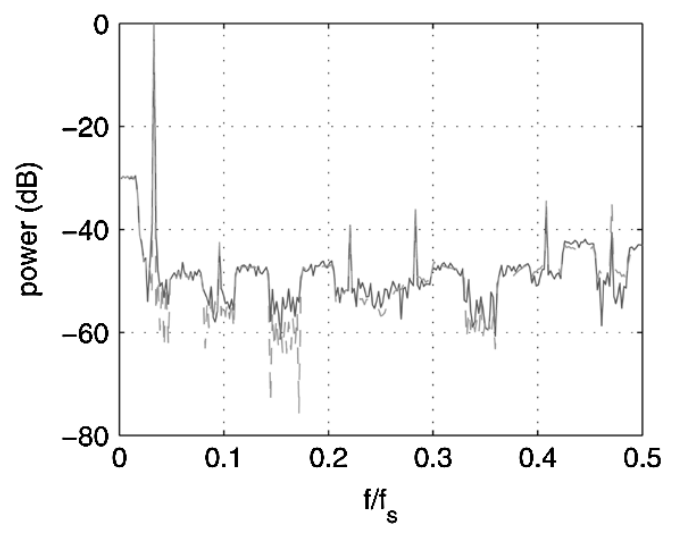

Fig. 2. Normalized spectrum of a 2.5 LSB sinusoid plus a (1/4) LSB noise with a correlation coefficient of 0.5 . The solid curve shows the spectrum obtained with a simulation model and the dashed curve shows the calculated spectrum.

frequencies which do not overlap before sampling but which do overlap after sampling, are summed. The derived model assumes sampling only after the multiplication. After integration and Fourier transformation, values for frequencies which overlap after sampling, are summed. These values represent power. So, in the case of the simulated spectrum, the amplitude of the sinusoidal signals with overlapping frequencies after sampling are summed while in case of the calculated spectrum only the power of these signals is summed. This explains the small difference. By calculating the spectra in case more noise is added to the channels of the cross correlator, the average distortion is decreased which is also known as the "dithering" effect (see, for example, [10]).

\section{CONCLUSION}

A general expression for the correlation function in the case of cross-correlating multibit quantized signals is determined for input signals consisting of partially correlated noise and a common periodic signal. Two extreme cases are: 1) the input signal consists of only noise and 2) the input signal consists of only a sinusoidal signal. For these cases, the results were already known and match with the developed expression. All other combinations of noise and periodic signals can also be analyzed using the general expression of the correlation function. In developing the model, the quantization staircase has been decomposed into a linear transfer function and an error-saw tooth. The effects of the error-saw tooth on the periodic signal are analyzed. The effects of the correlated and uncorrelated noise have been included. The well-known fact that noise smoothes the quantization staircase of an $A / D$ converter is demonstrated with the above model. Not included in the model is the effect of the uncorrelated noise and the sinusoidal signal on the SNR of the measured correlation function and the effects of the linearity errors of the A/D converters. These are subjects for further study.

\section{APPENDIX}

\section{A. General Expression of the Correlation Function}

The general expression for $R(t, \tau)$ with a periodic signal $f(t)$ can be written as

$$
\begin{aligned}
R(t, \tau)= & g^{2} f(t) f(t-\tau)+g^{2} \rho(\tau) \\
& +g f(t) \sum_{m_{2}=1}^{\infty} \frac{1}{m_{2} \pi} e^{-2 \pi^{2} g^{2} m_{2}^{2}} \cdot \sin \left(2 \pi m_{2} g f(t-\tau)\right) \\
& +g f(t-\tau) \sum_{m_{1}=1}^{\infty} \frac{1}{m_{1} \pi} e^{-2 \pi^{2} g^{2} m_{1}^{2}} \cdot \sin \left(2 \pi m_{1} g f(t)\right) \\
& +\sum_{m_{2}=1}^{\infty} 2 g^{2} \rho(\tau) e^{-2 \pi^{2} m_{2}^{2} g^{2}} \cdot \cos \left(2 \pi m_{2} g f(t-\tau)\right) \\
& +\sum_{m_{1}=1}^{\infty} 2 g^{2} \rho(\tau) e^{-2 \pi^{2} m_{1}^{2} g^{2}} \cdot \cos \left(2 \pi m_{1} g f(t)\right) \\
& +\sum_{m_{1}=1}^{\infty} \sum_{m_{2}=1}^{\infty} \frac{1}{m_{1} m_{2} \pi^{2}} e^{-2 \pi^{2}\left(m_{1}^{2}+m_{2}^{2}\right) g^{2}} \\
& \cdot\left[\sin \left(2 \pi m_{1} g f(t)\right) \sin \left(2 \pi m_{2} g f(t-\tau)\right)\right. \\
& \cdot \cosh \left(4 \pi^{2} g^{2} \rho(\tau) m_{1} m_{2}\right) \\
& +\cos \left(2 \pi m_{1} g f(t)\right) \cos \left(2 \pi m_{2} g f(t-\tau)\right) \\
& \left.\cdot \sinh \left(4 \pi^{2} g^{2} \rho(\tau) m_{1} m_{2}\right)\right]
\end{aligned}
$$

\section{REFERENCES}

[1] B. F. C. Cooper, "Correlators with two-bit quantization," Austral. J. Phy., vol. 23, pp. 521-527, 1970.

[2] F. K. Bowers and R. J. Klingler, "Quantization noise of correlation spectrometers," Astron. Astrophys. Suppl., vol. 15, pp. 373-380, 1974.

[3] R. A. Wannamaker, S. P. Lipshitz, J. Vanderkooy, and J. N. Wright, "A theory of nonsubtractive dither," IEEE Trans. Signal Processing, vol. 48, pp. 499-516, Feb. 2000.

[4] J. A. McFadden, "The correlation function of a sine wave plus noise after extreme clippings," IRE Trans. Inform. Theory, June 1956.

[5] W. R. Bennett, "Spectra of quantized signals," Bell Syst. Tech. J., vol. 27, pp. 446-472, July 1948.

[6] N. M. Blachman, "The inter-modulation and distortion due to quantization of sinusoids," IEEE Trans. Acoust., Speech, Signal Processing, vol. ASSP-33, pp. 1417-1426, Dec. 1985.

[7] M. Kendall and A. Stuart, The Advanced Theory of Statistics, 4th ed. London, U.K.: Griffin, 1977.

[8] L. Cheded and P. A. Payne, "The exact impact of amplitude quantization on multi-dimensional, high-order moments estimation," Signal Processing, vol. 39, no. 3, pp. 293-315, Sept. 1994.

[9] J. H. van Vleck and D. Middleton, "The spectrum of clipped noise," Proc. IEEE, vol. 54, Jan. 1966.

[10] A. W. Gunst, "The Use of Multi-Bit A/D Converters in Radio Astronomy," Univ. Twente, Enschede, The Netherlands, Report Code 060.2721, June 1999 . 